\title{
SOBRE A IDADE DAS ÁGUAS SUBTERRÂNEAS NO POLÍGONO DAS SECAS DO NORDESTE BRASILEIRO*
}

\author{
M. A. GEYH** e K. KREYSIN(;***
}

\begin{abstract}
Residence times and radiocarbon model ages were obtained on intensively mineralized ground waters of Northeast Brazil, by means of $\mathrm{C}^{14}$ and $\mathrm{H}^{3}$ analyses. The results show a close correlation between the geologic setting and ground water renowal. The older waters are found only on joint systems. For other situations the wells show reduced content of captation waters.

Initial concentration of $\mathrm{C}^{14}$ vary between 75 and $100 \%$ modern, reflecting differences on the basement geology, which is composed by gneisses and mica schists. Residence times and total mineral content of the ground waters seem to bear a normal relationship.
\end{abstract}

INTRODUC̣ÃO As idéias sobre idades das águas subterrâneas salinizadas no embasamento cristalino do Nordeste do Brasil (Schoff) carecem de uma revisão face aos mais novos conhecimentos no campo da Hidrogeologia. A aplicação bem sucedida dıs métodos do $\mathrm{C}^{14}$ e $\mathrm{H}^{3}$ em águas cársticas na determinação dos tempos de residência (TR) (Geyh \& Mairhofer) sugeriu a utilização desses métodos nas pesquisas das áreas de trabalho da MGA, não obstante a diversidade da geologia. Segundo as reflexões hidrogenéticas agora elaboradas e baseadas em numerosas análises hidroquímicas, a hipótese da existência de águas fósseis conatas não mais parece plausível. Supõe-se uma água subterrânea mais jovem, que sofre uma contínua renovação. Sobre as causas da alta salinidade dessas águas, ainda não foi possível estabelecer uma hipótese bem fundamentada.

Situação hidrogeológica da área em estudo A geologia da área em estudo se caracteriza como embasamento cristalino com zonas de cisalhamento tectônico. Orograficamente trata-se de um peneplano com Inselberge isolados. Predomina uma floresta arbustiva, resistente às secas. Os poços se localizam, na sua maioria, em vales de riachos intermitentes no peneplano e produzem, em $90 \%$ dos casos, águas com alto teor salino. O clima é semi-árido, com uma taxa de precipitação de 300 a $400 \mathrm{~mm} /$ ano. Para a renovação da água subterrânea, contribuem, principalmente, as chuvas torrenciais de meados de dezembro a abril. A temperatura média anual é de $27,5^{\circ} \mathrm{C}$ e a taxa de evaporação teórica, segundo experiências em laboratório, é de $2000 \mathrm{~mm}$ por ano, aproximadamente.

A reserva útil de água subterrânea ocorre em dois níveis, sendo que o superior, o principal fornecedor de água potável, limita-se aos aluviões fluviais, com espessuras de 2 a $3 \mathrm{~m}$, e pode se esgotar nos períodos de seca extrema. As águas fortemente salinizadas dos poços tubulares provêm do segundo nível, localizado nas zonas cisalhadas e diaclasadas do embasamento cristalino. A renovação da água subterrânea também se verifica através das diáclases e zonas de cisalhamento.

Tempo de residência de água e idade-modelo $\mathrm{C}^{14}$ A idade-modelo $\mathrm{C}^{14}$, calculada esquematicamente, de uma água subterrânea, quando esta pertence a um sistema fechado com escoamento homogêneo, corresponde, em geral, a sua idade real quando

* Sob os auspícios da Superintendência do Desenvolvimento do Nordeste. Depto. de Recursos Naturais. Divisão de Hidrogeologia

**Bundesanstalt für Bodenforschung

***Missão Geológica Alemã no Brasil 
levado em conta um coeficiente de ajustamento (fator de diluição). No entanto, quando se trata de águas que ainda se localizam na zona de influência da própria área de realimentação, deve ser levada em conta a sua composição, na qual as componentes de idade variável diminuem exponencialmente com o aumento da distância à área de realimentação. Por esse motivo, em lugar dos dados de idade usuais para as águas que ainda não se separaram da área de realimentação, é preferível o emprego do conceito de tempo de residência $(T R)$, que pode ser avaliado com auxílio da determinação dos teores de $\mathrm{C}^{14}$ e $\mathrm{H}^{3}$. É necessário, contudo, que se conheça a influência das experiências nucleares sobre o ciclo da concentração de $\mathrm{C}^{34}$ no $\mathrm{CO}_{2}$ da atmosfera e do teor de $\mathrm{H}^{3}$ nas precipitações pluviométricas da área em estudo (Geyh, 1972).

O conhecimento do tempo de residência $(T R)$ possibilita, então, entre outros fatores, a determinação do tempo de renovação da reserva potencial de uma área de realimentação ou da reserva potencial de água subterrânea de uma mesma área de realimentação. Ao mesmo tempo, permite determinar a sua capacidade quando é conhecida a descarga anual natural como, por exemplo, em fontes naturais.

A verificação do fato de a maioria das águas subterrâneas estudadas ainda localizadas nas áreas de realimentação satisfazerem ao "modelo exponencial" (Eriksson, 1962) tem conseqüências para a interpretação dos resultados de "Estudos de Tracer":

1) Ao contrário das suposições levantadas por Campos (1970), a partir do teor em $\mathrm{H}^{3}$ de amostras isoladas não é possível a determinação nem de sua idade nem das cotas de afluxo de componentes recentes. Para tanto necessitamse, ainda, de pelo menos análises de $\mathrm{C}^{14}$. Campos supôs, erroneamente, que águas com menos de $2 T E$ fossem mais velhas que 15 anos; com 2 a $11 T E$, tivessem de 5 a 15 anos; e, com mais de $11 T E$, fossem mais novas que 5 anos.

2) Como as águas subterrâneas ainda não isoladas da sua área de realimentação sempre contêm uma parte recente, independente de seu $T R$, experiências de corantes permitem apenas conclusões sobre comunicações hidráulicas, mas não sobre características da água em si.

Resultados das análises de $\mathrm{C}^{14}$ e $\mathrm{H}^{3}$ Os resultados das análises de $\mathrm{C}^{14}$ e $\mathrm{H}^{3}$, os valores de $\mathrm{pH}$ e os teores salinos totais das amostras de água examinadas estão relacionados na Tab. I, juntamente com resultados interpretativos intermediários, as idades-modelo $\mathrm{C}^{14}$ calculadas e os tempos de residência $(T R)$. Para a interpretação, foram usadas as curvas de Nydal e Loveseth (1970) para o Hemisfério Meridional, que reflete a variação do teor de $\mathrm{C}^{14}$ no $\mathrm{CO}_{2}$ do ar com o tempo. A curva de referência dos teores de $\mathrm{H}^{3}$ foi reproduzida na Fig. 1. Foi elaborada com base em determinações de trítio em amostras pluviais do Rio de Janeiro e Nova Zelândia (IAEA, 1967). Os autores não desconhecem a insegurança do resultado, condicionada, principalmente, à grande variação das concentrações do $\mathrm{H}^{3}$ das chuvas tropicais. Os resultados mais recentes de análises de $\mathrm{H}^{3}$ em amostras pluviais na região de Petrolina (Campos, 1970) mostram, todavia, que a curva $\mathrm{H}^{3}$ da Fig. 1 aproxima-se bastante da real.

Para a transformação dos teores de $\mathrm{G}^{14}$ em tempos de residência, foram tomadas como base variadas concentrações iniciais $(75,85,100 \%$ moderno). A multiplicidade de valores $T R$ daí resultante é eliminada quando são usados simultaneamente medidas de $\mathrm{H}^{3}$ de amostras de diferentes anos (1968 e 1969) e quando então se verifica a coincidência de valores isolados. Dessa maneira, determinam-se também as concentraç̃es iniciais de $\mathrm{C}^{14}$. 
Tabela I

\begin{tabular}{|c|c|c|c|c|c|c|c|c|c|c|c|c|c|c|c|c|c|c|c|}
\hline \multirow{4}{*}{\begin{tabular}{r|}
$\mathrm{N} 0$ \\
9 \\
10
\end{tabular}} & \multirow{4}{*}{$\begin{array}{l}\text { NO ME } \\
\text { ALTOS } \\
\text { TANQUINHO }\end{array}$} & \multirow{4}{*}{\begin{tabular}{|l}
$\mathrm{PH}$ \\
7,8 \\
6,5
\end{tabular}} & \multirow{2}{*}{\multicolumn{2}{|c|}{$\begin{array}{c}\mathrm{HCO}_{3} \text { Teor miner. } \\
\mathrm{mg} / \mathrm{l}\end{array}$}} & \multirow{2}{*}{$\mathrm{mm}$} & \multirow{2}{*}{\multicolumn{2}{|c|}{\begin{tabular}{|l|l|} 
3 H-Conc.T.E. \\
1968 & 1969 \\
\end{tabular}}} & \multicolumn{2}{|c|}{${ }^{14} \mathrm{c}-$ concf $\left.\% \mathrm{~m}\right)$} & \multicolumn{2}{|c|}{3 H-T.R. (a) } & \multicolumn{3}{|c|}{${ }^{14} \mathrm{C}-$ T. R. (1968) } & \multirow{2}{*}{\multicolumn{2}{|c|}{$\begin{array}{c}{ }^{14} \mathrm{C}-\text { T.R. } \\
75 \% \mid 85 \%\end{array}$}} & \multirow{2}{*}{$\begin{array}{l}(1969) \\
100 \%\end{array}$} & \multirow{2}{*}{$\left|\begin{array}{l}\text { He-Conc inic } \\
\% \text { moderno }\end{array}\right|$} & \multirow{2}{*}{$\begin{array}{c}\text { T.R. (a) } \\
{ }^{14} \mathrm{C} \text { :dad.mod.(o.ar) }\end{array}$} \\
\hline & & & & & & & & 1968 & 1969 & 1968 & 1869 & $75 \%$ & $85 \%$ & |100\% | & & & & & \\
\hline & & & 10,8 & 1300 & & $<2,6$ & 2,3 & 63,2 & 71,1 & $>80$ & $>80$ & $>100$ & $>100$ & $>100$ & $>50$ & $>100$ & $>100$ & $100 ?$ & 2740 \\
\hline & & & 17,2 & 3900 & 5,5 & $\mid<1,4$ & $<1,4$ & & 85,0 & $>100$ & $>100$ & & & & 30 & 80 & $>100$ & 100 & 1300 \\
\hline 57 & PITOMBEIRO & 7,1 & 22,1 & 5800 & 8 & & $<2,3$ & & 89,5 & & $>80$ & & & & 9 & 20 & $>100$ & 100 & 900 \\
\hline 30 & sítio Novo & 6,7 & 15,9 & 7300 & 8 & $<2,6$ & $<0,7$ & & 89,7 & $>80$ & $>100$ & & & & 20 & 60 & $>100$ & 100 & 880 \\
\hline 92 & JACARÉ & 7,2 & 13,0 & 1500 & 5 & & $<1,1$ & & 89,8 & & $>100$ & & & & 20 & 60 & $>100$ & 100 & 860 \\
\hline 72 & BARRA DO RUSSO & & & & & $|<2,6|$ & & 90,4 & & $>80$ & & 19 & 40 & 100 & & & & 100 & 820 \\
\hline 4 & BRUNNEN $\mathrm{km} 25$ & & & & & $\mid<2,4$ & & 91,8 & & $>80$ & & 17 & 40 & 100 & & & & 100 & 800 \\
\hline 94 & PISARA & 6,8 & 12,6 & 3400 & 5 & & 2,1 & & 86,2 & & $>80$ & & & & 25 & 80 & $>100$ & 100 & 80 \\
\hline 84 & PITANGA & 6,6 & 11,5 & 5100 & 7 & & 2,5 & & 89,5 & & 80 & & & & 25 & 60 & $>100$ & 100 & 80 \\
\hline 44 & CARETÃO & & & & & 2,6 & & 101,7 & & 80 & & 10 & 20 & 90 & & & & 100 & 80 \\
\hline 28 & URUAS & 7,0 & $\mid 1,1$ & 2300 & 4 & & 2,9 & & 105,1 & & 80 & & & & 9 & 20 & 60 & 100 & 70 \\
\hline 58 & MELANCIA & & & & & 4,8 & & 93,7 & & 60 & & 15 & 35 & 75 & & & & 95 & 70 \\
\hline 76 & DORMENTE & & & & & $\mid<1,7$ & & 94,5 & & $>100$ & & 15 & 30 & 60 & & & & $100 ?$ & 70 \\
\hline 88 & $\angle A G O A$ DE FORA & 6,7 & 12,6 & 3500 & 5 & & $<1,3$ & & 104,6 & & $>100$ & & & & 9 & 20 & 60 & 100 & 60 \\
\hline 12 & ANGICAL & 6,5 & 9,0 & 1000 & 4 & & 14,8 & & 134,9 & & 20 & & & & & 3 & 11 & 100 & 15. \\
\hline 67 & PALÁCIO & 6,5 & 24,5 & 10.500 & 10 & 2,8 & 1,8 & 101,2 & 82,3 & 80 & $>100$ & 10 & 20 & 90 & 40 & 100 & $>100$ & 90 & 90 \\
\hline 50 & PRENSA & 7,5 & 25,7 & 3350 & 5 & & 4,6 & & 82,6 & & 60 & & & & 40 & 100 & $>100$ & 80 & 60 \\
\hline 17 & CAMPO LIMPO & 7,0 & 14,3 & 1470 & 3 & & 5,3 & & 90,4 & & 60 & & & & 25 & 60 & $>100$ & 85 & 60 \\
\hline 204 & CAPIM III $(4,7)$ & 6,5 & 4,3 & 400 & 12 & & 9,8 & & 90,3 & & 30 & & & & 20 & 50 & $>100$ & 80 & 40 \\
\hline 204 & CAPIM III $(3,3)$ & 6,5 & & & 12 & & 6,1 & & & & 50 & & & & & & & & $50 ?$ \\
\hline 204 & CAPIM III $(4,7)$ & 6,5 & & & 12 & & 9,5 & & 89,8 & & 30 & & & & 20 & 50 & $>100$ & 80 & 40 \\
\hline 59 & LAGOA GRANDE & 7,4 & 7,8 & 2900 & 4 & & 7,8 & & 89,5 & & 40 & & & & 20 & 60 & $>100$ & 80 & 40 \\
\hline 8 & CAEIRA & & & & & 9,4 & & 91,1 & & 35 & & 18 & 45 & 100 & & & & 80 & 40 \\
\hline 42 & CAISARA II & & & & & & 12,0 & & & & 25 & & & & & & & & $25 ?$ \\
\hline 40 & PASTOS BONS & & & & & $<2,5$ & & & & 80 & & & & & & & & & $>80 ?$ \\
\hline 1 & LAGOA GRANDE & 7,0 & 18,9 & 900 & 3 & & 4,4 & & 78,3 & & 70 & & & & 60 & $>100$ & $>100$ & 80 & 60 \\
\hline 1 & LAGOA GRANDE & & & & & $\mid<2,7$ & 3,0 & & 78,3 & & 90 & & & & 60 & 100 & $>100$ & 85 & 90 \\
\hline 25 & CURUPAITÉ & 7,0 & 17,7 & 4000 & 4 & & 5,0 & & 89,1 & & 60 & & & & 25 & 60 & $>100$ & 85 & 60 \\
\hline 46 & CAL & 7,2 & 15,7 & 2050 & 4 & & & & 88,8 & & & & & & 25 & 60 & $>100$ & $\leq 80$ & $\leq 25$ \\
\hline
\end{tabular}




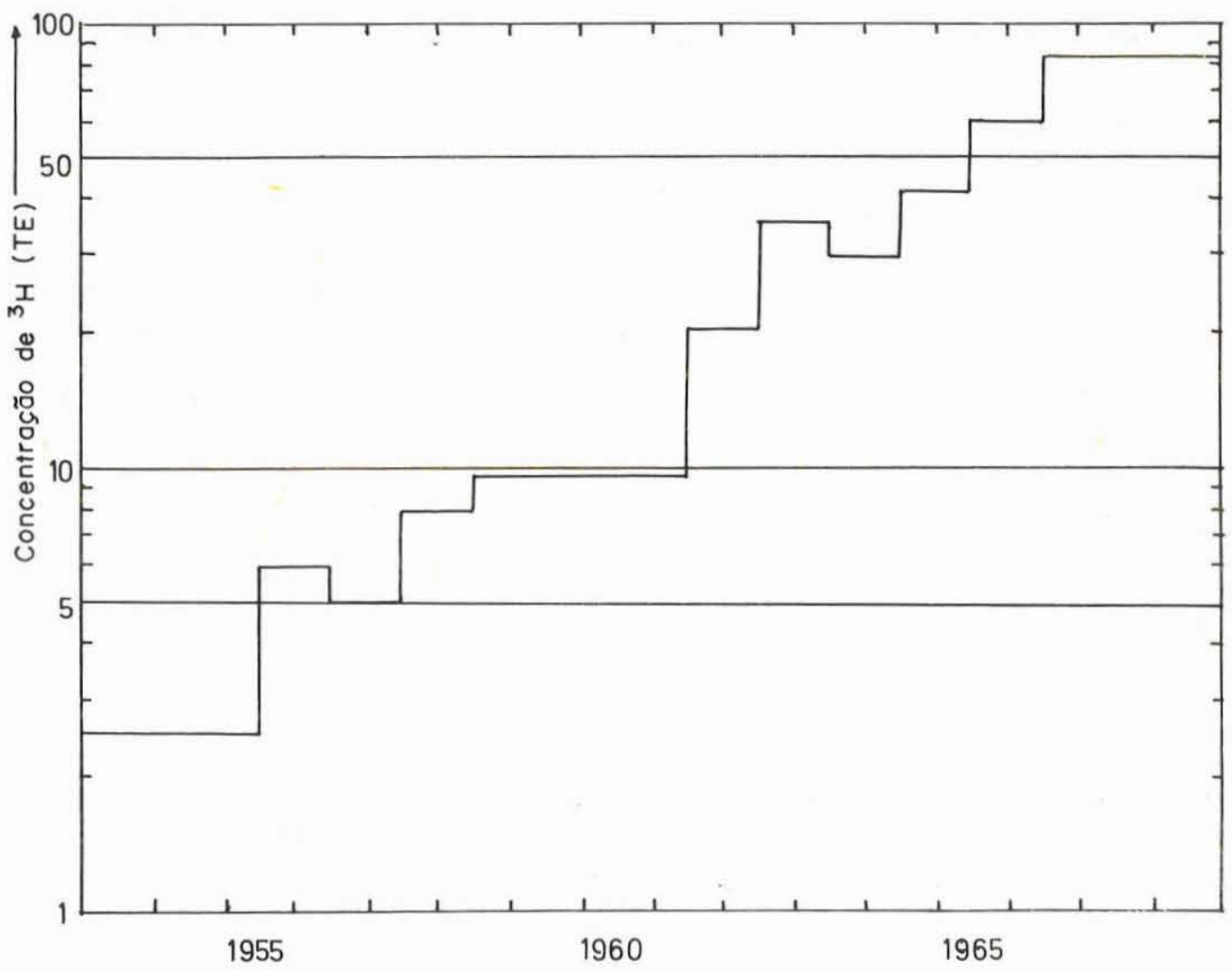

Figura 1 - Concentração média de ${ }^{3} \mathrm{H}$ das chuvas de setembro a março nos anos 1953-1969

Concentrações iniciais de $\mathrm{C}^{14}$ da água subterrânea As concentrações iniciais de $\mathrm{C}^{14}$ das águas subterrâneas ou fator de diluição da área de estudos de Petrolina envolve um campo de valores desconhecido em sua amplitude. Varia de 80 a $100 \%$ moderno, ultrapassando, portanto, o valor comum de $85 \%$ (Vogel, 1970).

Esse fato se explica com a origem dos carbonatos no cristalino como produtos recentes e sub-recentes da alteração de feldspato sob ação de $\mathrm{CO}_{2}$.

Como o teor de $\mathrm{C}^{14}$ correspondente passa para a fração de carbonato, nessas condições a concentração inicial de $\mathrm{C}^{14}$ das águas subterrâneas pode atingir até a $100 \%$ moderna (Geyh, 1970).

Os resultados das pesquisas refletem as interpretações técnicas. Nas zonas do cristalino antigo maciço e dos gnaisses, assim como da zona de falhamento N-S a Oeste, observam-se as concentrações de $\mathrm{C}^{14}$ mais elevadas. Isso significa que todo o carbonato formado através de intemperismo é imediatamente absorvido com a neo-formação de água subterrânea. Em contraposição, na área de extensão do micaxisto, devem ser encontrados feldspatos alterados sub-recentemente cuja fração carbonática tem idade de alguns milhares de anos.

Geologia - tempos de residência e idades-modelo $C^{14}$ Verifica-se a correlação presumível, com base nas experiências adquiridas, entre a geologia das áreas de realimentação e as idades-modelo de $\mathrm{C}^{14}$, ou seja, os tempos de residência das respectivas águas subterrâneas. Nas zonas de falhamento N-S que ocorrem a Oeste, parece existirem reservatórios, podendo ocorrer, portanto, águas mais idosas. A estas pertencem as amostras 
Figura 2 - Relação entre o tempo de residência e o teor mineral

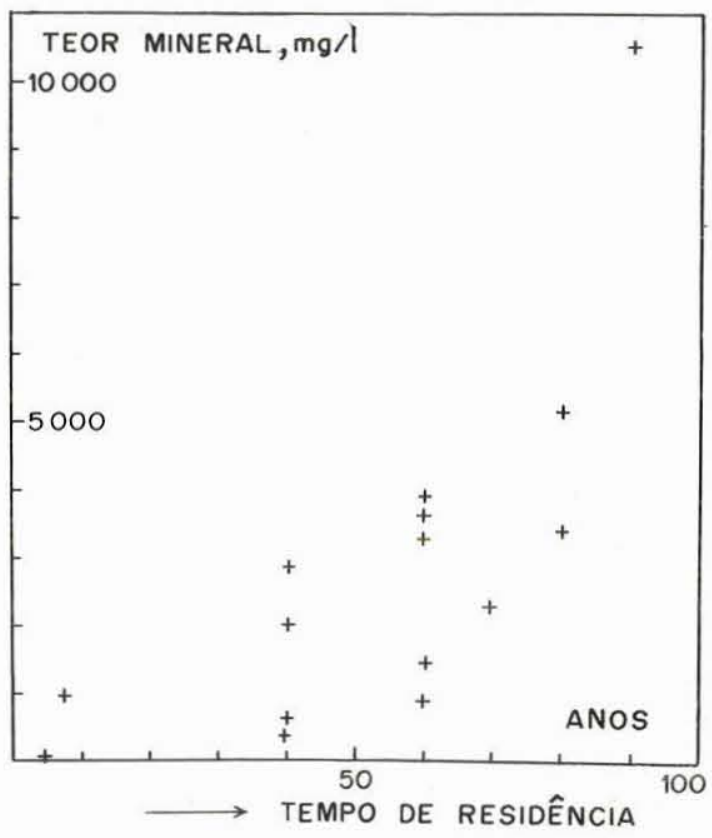

de Altos (n. ${ }^{\circ}$ ) e Tanquinho (n. $\left.{ }^{\circ} 10\right)$, cujas idades-modelo são, no mínimo, de algumas centenas, até, no máximo, de 2700 anos. Dos dados de isótopos, é impossível concluir se as duas amostras pertencem ao mesmo sistema hidráulico. Não se conhece a localização da área de realimentação dessas águas.

Nas zonas de cristalino maciço ou de gnaisses diaclasados nas partes meridional e setentrional da área estudada, também ocorrem águas idosas, se bem que mais jovens do que as descritas no parágrafo anterior. É possível que exista, pelo menos para alguns poços meridionais (Pitombeira n. ${ }^{\circ}$ 57, Sítio Novo n. ${ }^{\circ} 4$, Jacaré n. ${ }^{\circ}$ 91, Banco Russo n. 72 e Poço km 25 n. ${ }^{\circ}$ ), uma comunicação hidráulica com o Rio São Francisco, de acordo com a posição do nível freático desses poços. A idade-modelo $\mathrm{C}^{14}$ elevada indica a existência de espaço útil para armazenamento. Entretanto nem todas as amostras examinadas seguem esse modelo, por exemplo, as amostras de Piçarra (n. $\left.{ }^{\circ} 94\right)$, Pitanga (n. $\left.{ }^{\circ} 84\right)$, Carretão (n. $\left.{ }^{\circ} 44\right)$, Uruás (n. ${ }^{\circ}$ 28), Melancia (n. ${ }^{\circ}$ 58), Dormente (n. $\left.{ }^{\circ} 76\right)$, Lagoa da Fossa (n. $\left.{ }^{\circ} 88\right)$ e Angical (n. ${ }^{\circ}$ 12). Nesses casos, trata-se de sistemas de diáclases muito limitados. Essa limitação causa as variações do tempo de residência e das características químicas das águas.

A amostra Palácio n. ${ }^{\circ} 67$ tem uma posição geológica intermediária entre as anteriores e as das águas dos micaxistos (Prensa n. ${ }^{\circ}$ 50; Campo Limpo n. ${ }^{\circ} 17$; Capim n. ${ }^{\circ}$ 204; Lagoa Grande n. ${ }^{\circ}$ 59; Caiçara n. ${ }^{\circ} 8$; Caiçara II n. ${ }^{\circ} 42$; Pastos Bons n. ${ }^{\circ} 40$; Lagoa Grande n. ${ }^{\circ} 1$ e Curupaité n. ${ }^{\circ}$ 25), estas com concentrações iniciais de $\mathrm{C}^{14}$ reduzidas. Isso é confirmado também pelo fator de dissolução de 0,90 daquela amostra. Nos outros casos, faltam sistemas fechados de diáclases mais extensos, o que explica os tempos de residência reduzidos.

A amostra Cal n. ${ }^{\circ} 46$, oriunda de um poço de uma lente de calcário com reservatório fechado, isolado do micaxisto, representa uma exceção. Como a amostra para $\mathrm{H}^{3}$ foi extraviada durante o transporte, não foi possível, infelizmente, a determinação da concentração inicial de $\mathrm{C}^{14}$. Com base nas experiências adquiridas na Europa Central, pode-se supor um valor de $75 \%$ moderno, de modo que o tempo de residência correspondente é menor que 25 anos. 


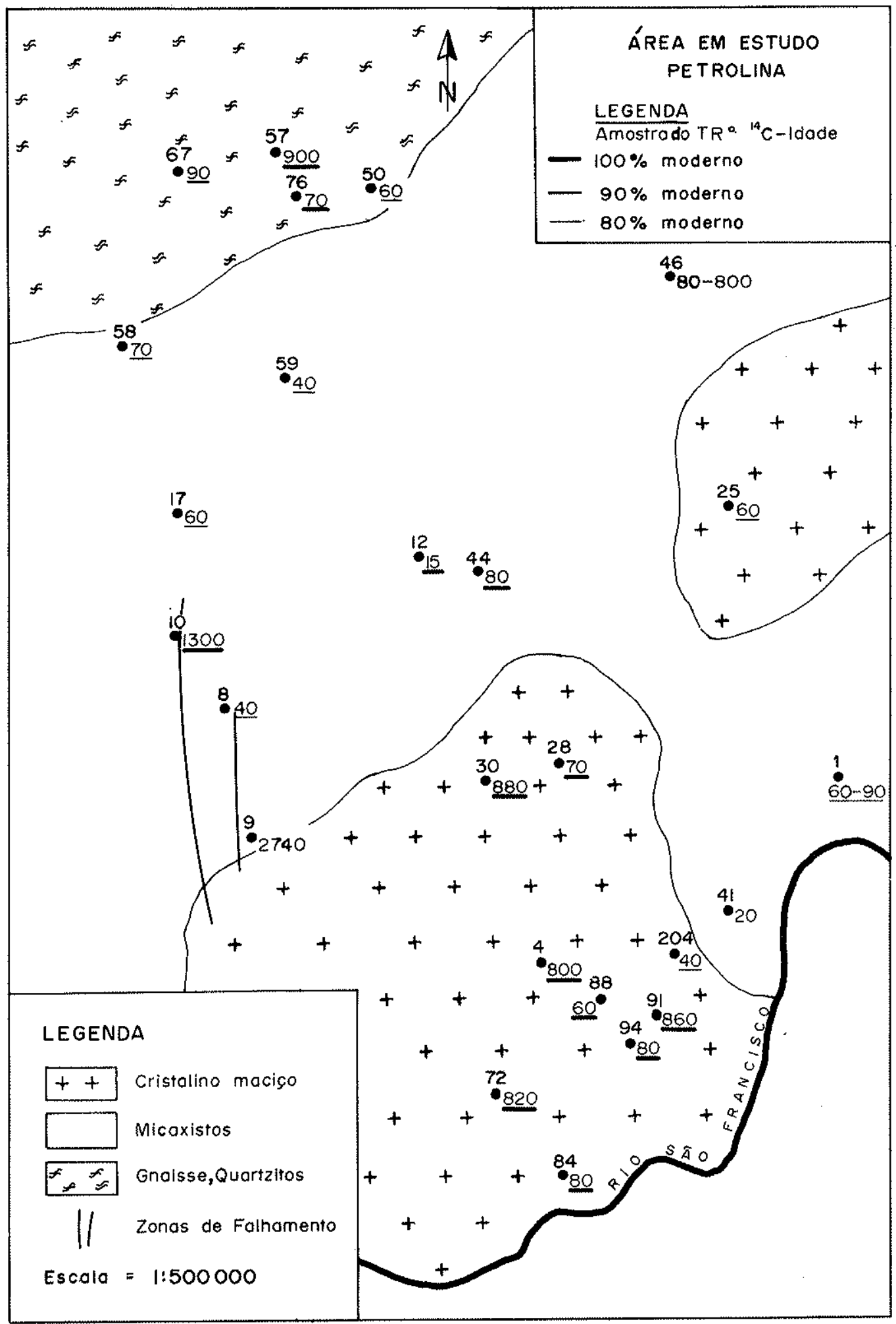

Figura 3 - Área em estudo 
Teor mineral e tempo de residência das águas subterrâneas Não é possível verificar uma relação entre os tempos de residência e a química do ácido carbônico, ou seja, valores de $\mathrm{pH}$, em contraposição ao teor mineral. Como a área de captação das águas subterrâneas jovens com grandes tempos de residência e mais distante que a das com pequenos tempos de residência, é compreensível uma elevação do teor mineral com o aumento da componente de águas idosas. O fato de isso não ocorrer com as águas mais idosas explica-se pelas condições favoráveis de regeneração, em rochas mais intensamente diaclasadas, pelo menos em alguns casos.

\section{BIBLIOGRAFIA}

CAMPOS, M. M. - 1970 - Levantamento preliminar de teores de trítio em águas do Nordeste Brasileiro. Instituto de Pesquisas Radioativas UFMG. CNEN.

ERIKSSON, E. - 1962 - Radioactivity in Hydrology, in: Nuclear Radiation in Geophysics, 42, ed. H. Israel \& A. Krebs, Springer Verlag.

GEYH, M. A. - 1970 - Carbon-14 concentration of lime in soils and aspects of the carbon-14 dating of groundwater. Isotope Hydrology Viena, IAEA, pp. 215-224.

GEYH, M. A. - 1971 - Die ${ }^{14} \mathrm{C}$-und ${ }^{3} \mathrm{H}$-Methode in der angewandten Hydrogeologie. Wasserkalender, 1972, DZW Dusseldort, im Druck.

GEYH, M. A. e MAIRHOFER, J. - 1970 - Der naturliche Carben-14 und Tritium-Gehalt. Steirische Beitrage zur Hydrogeologie, 22: 63-81.

GEYH, M. A. c MAIRHOFER, J. - 1972 - Determination of the dilution factor of groundwater. Radiocarbon Dating Conference, Nova Zelândia, no prelo.

IAEA - 1967 - Technical Report Series: Tritium and Other Environment Isotopes in the Hydrological Cycle, Viena, 73.

KREYSING, K. LENZ, R. - 1969 - Hydrogeologischer Teil des Jahresberichtes 1968 der DGM in Brasilien, pp. 38-58, inédito.

NYDAL, R. e LOVESETH, K. - 1970 - Prospective Decrease in Atmospheric Radiocarbon, J. Geophys. Res. 75: 2 271-2 278.

VOGEL, J. C. - 1970 - Carbon-14 dating of groundwater. Isotope Hydrology, IAEA. Viena, pp. 225-240. 